\title{
Less is More: Micro-expression Recognition from Video using Apex Frame
}

\author{
Sze-Teng Liong ${ }^{\mathrm{a}}$, John See ${ }^{\mathrm{b}}$, KokSheik Wong ${ }^{\mathrm{c}, *}$, Raphael C.-W. Phan ${ }^{\mathrm{d}}$ \\ ${ }^{a}$ Department of Electronic Engineering, Feng Chia University, Taichung 40724, Taiwan R.O.C. \\ ${ }^{b}$ Faculty of Computing and Informatics, Multimedia University, 63100 Cyberjaya, Malaysia \\ ${ }^{c}$ School of Information Technology, Monash University Malaysia, 47500 Selangor, Malaysia \\ ${ }^{d}$ Faculty of Engineering, Multimedia University, 63100 Cyberjaya, Malaysia
}

\begin{abstract}
Despite recent interest and advances in facial micro-expression research, there is still plenty of room for improvement in terms of micro-expression recognition. Conventional feature extraction approaches for micro-expression video consider either the whole video sequence or a part of it, for representation. However, with the high-speed video capture of microexpressions (100-200 fps), are all frames necessary to provide a sufficiently meaningful representation? Is the luxury of data a bane to accurate recognition? A novel proposition is presented in this paper, whereby we utilize only two images per video, namely, the apex frame and the onset frame. The apex frame of a video contains the highest intensity of expression changes among all frames, while the onset is the perfect choice of a reference frame with neutral expression. A new feature extractor, Bi-Weighted Oriented Optical Flow (Bi-WOOF) is proposed to encode essential expressiveness of the apex frame. We evaluated the proposed method on five micro-expression databases- CAS(ME) ${ }^{2}$, CASME II, SMIC-HS, SMIC-NIR and SMIC-VIS. Our experiments lend credence to our hypothesis, with our proposed technique achieving a state-of-the-art F1-score recognition performance of 0.61 and 0.62 in the high frame rate CASME II and SMIC-HS databases respectively.
\end{abstract}

Keywords: Micro-expressions, emotion, apex, optical flow, optical strain, recognition

\section{Introduction}

Have you ever thought that someone was lying to you, but have no evidence to prove it? Or have you always found it difficult to interpret one's emotion? Recognizing micro-expressions could help to solve these doubts.

Micro-expression is a very brief and rapid facial emotion that is provoked involuntarily [1], revealing a person's true feelings. Akin to normal facial expression, also known as macro-expression, it can be categorized into six basic emotions: happy, fear, sad, surprise, anger and disgust. However, macro-expressions are easily identified in realtime situations with the naked eye as it occurs between 2-3 seconds and can be found over the entire face region. On the other hand, a micro-expression is both micro (short duration) and subtle (small intensity) [2] in nature. It lasts between $1 / 5$ to $1 / 25$ of a second and usually occurs in only a few parts of the face. These are the main reasons why people are sometimes unable to realize or recognize the genuine emotion shown on a person's face [3, 4]. Hence, the ability to recognize micro-expressions is beneficial in both our mundane lives and also society at large. At a personal level, we can differentiate if someone is telling the truth or

\footnotetext{
* Corresponding author

Email addresses: christyliong91@gmail.com (Sze-Teng Liong), johnsee@mmu.edu.my (John See), wong.koksheik@monash.edu (KokSheik Wong), raphael@mmu.edu.my (Raphael C.-W. Phan)
}

lie. Also, analyzing a person's emotions can help facilitate understanding of our social relationships, while we are increasingly awareness of the emotional states of our own selfs and of the people around us. More essentially, recognizing these micro-expressions is useful in a wide range of applications, including psychological and clinical diagnosis, police interrogation and national security [5-7].

Micro-expression was first discovered by psychologists, Ekman and Friesen [1] in 1969, from a case where a patient was trying to conceal his sad feeling by covering up with smile. They detected the patient's genuine feeling by carefully observing the subtle movements on his face, and found out that the patient was actually planning to commit suicide. Later on, they established Facial Action Coding System (FACS) [8] to determine the relationship between facial muscle changes and emotional states. This system can be used to identify the exact time each action unit (AU) begins and ends. The occurrence of the first visible $\mathrm{AU}$ is called the onset, while that of the disappearance of the AU is the offset. Apex is the point when the $\mathrm{AU}$ reaches the peak or the highest intensity of the facial motion. The timings of the onset, offset and apex for the AUs may differ for the same emotion type. Figure 1 shows a sample sequence containing frames of a surprise expression from a micro-expression database, with the indication of onset, apex and offset frames. 


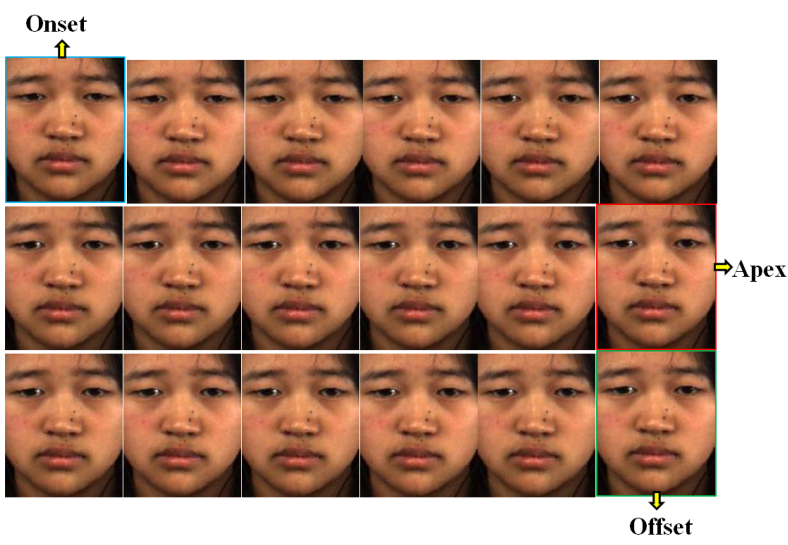

Figure 1: Example of a sequence of image frames (ordered from left to right, top to bottom) of a surprise expression from the CASME II [9] database, with the onset, apex and offset frame indications

\section{Background}

Micro-expression analysis is arguably one of the lesser explored areas of research in the field of machine vision and computational intelligence. Currently, there are less than fifty micro-expressions related research papers published since 2009. While databases for normal facial expressions are widely available [10], facial micro-expression data, particularly those of spontaneous nature, is somewhat limited for a number of reasons. Firstly, the elicitation process demands for good choice of emotional stimuli that has high ecological validity. Post-capture, the labeling of these micro-expression samples require the verification of psychologists or trained experts. Early attempts centered on the collection of posed micro-expression samples, i.e. USF-HD [11] and Polikovsky's [12] databases, which went against the involuntary and spontaneous nature of micro-expressions [13]. Thus, the lack of spontaneous micro-expression databases had hindered the progress of micro-expression research. Nonetheless, since 2013, the emergence of three prominent spontaneous facial microexpression databases - the SMIC from University of Oulu [14] and the CASME/ CASME II/ CAS(ME) ${ }^{2}[9,15,16]$ from the Chinese Academy of Sciences, have breathed fresh interest into this domain.

There are two primary tasks in an automated microexpression system, i.e., spotting and recognition. The former identifies a micro-expression occurrence (and its interval of occurrence), or to locate some important frame instances such as onset, apex and offset frames (see Figure 1). Meanwhile, the latter classifies the expression type given the "spotted" micro-expression video sequence. A majority of works focused solely on the recognition task of the system, whereby new feature extraction methods have been developed to improve on micro-expression recognition rate. Figure 2 illustrates the optical flow magnitude and optical strain magnitude computed between the onset (assumed as neutral expression) and subsequent frames. It is observed that the apex frames (middle and bottom

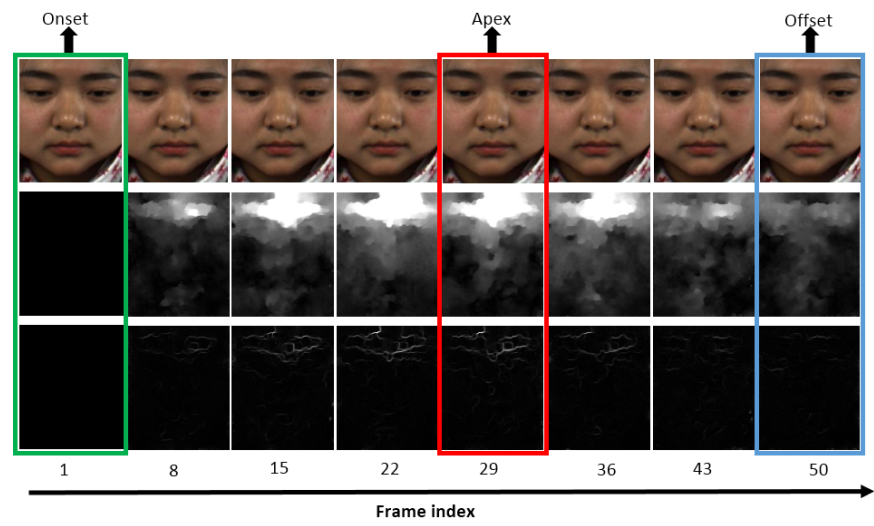

Figure 2: Illustration of (top row) original images; (middle row) optical flow magnitude computed between the onset and subsequent frames; and (bottom row) optical strain computed between the onset and subsequent frames

rows in Figure 2) are the frames with the highest motion changes (bright region) among the video sequence.

Micro-expression databases are pre-processed before releasing to the public. This process includes face registration, face alignment and ground-truth labeling (i.e., AU, emotion type, frame indices of onset, apex and offset). In the two most popular spontaneous micro-expression databases, namely the CASME II [9] and SMIC [14], the first two processes (face registration and alignment) were achieved automatically. Active Shape Model (ASM) [17] is used to detect a set of facial landmark coordinates; then the faces are transformed based on the template face according to its landmark points using the classic Local Weighted Mean (LWM) [18] method. However, the last process, i.e., ground-truth labeling, is not automatic and requires the help of psychologists or trained experts. In other words, the annotated ground-truth labels may vary depending on the coders. As such, the reliability and consistency of the markings are less than ideal, which may affect the recognition accuracy of the system.

\subsection{Micro-expression recognition}

Recognition baselines for the SMIC, CASME II and $\mathrm{CAS}(\mathrm{ME})^{2}$ databases were established with the original works $[9,14,16]$ with Local Binary Patterns-Three Orthogonal Planes (LBP-TOP) [19] as the choice of spatio-temporal descriptor, and Support Vector Machines (SVM) [20] as classifier. Subsequently, a number of LBP variants [21-23] were proposed to improve on the usage of LBP-TOP. Wang et al. [21] presented an efficient representation that reduces the inherent redundancies within LBP-TOP, while Huang et al. [22] adopted an integral projection method to boost the capability of LBP-TOP by supplementing shape information. More recently, another LBP variant called SpatioTemporal Completed Local Quantization Pattern (STCLQP) [23] was proposed to extract three kinds of information (local sign, magnitude, orientation) before encoding them into a compact codebook. A few works stayed away from using conventional 
pixel intensity information in favor of other base features such as optical strain information $[24,25]$ and monogenic signal components [26], before describing them with LBPTOP. There were other methods proposed that derived useful features directly from color spaces [27] and optical flow orientations [28].

Two most recent works $[29,30]$ presented alternative schemes to deal with the minute changes in microexpression videos Le et al. [29] hypothesized that dynamics in subtle occurring expressions contain a significantly large number of redundant frames, therefore they are likely to be "sparse". Their approach determines the optimal vector of amplitudes with a fixed sparsity structure and recognition performance reportedly significantly better than using the standard Temporal Interpolation Model (TIM) [31]. Xu et al. [30] characterized the local movements of a micro-expression by the principal optical flow direction of spatiotemporal cuboids extracted at a chosen granularity. On the other hand, the works by $[32-34]$ reduce the dimensionality of the features extracted from micro-expression videos using Principal Component Analysis (PCA), while [35] employed sparse tensor analysis to minimize the dimension of features.

\subsection{Micro-expression spotting}

There are several works which attempted to spot the temporal interval (i.e., onset-offset) containing microexpressions from raw videos in the databases. By raw, we refer to video clips in its original form, without any pre-processing. In [36], the authors searched for the frame indices that contain micro-expressions. They utilized ChiSquared dissimilarity to calculate the distribution difference between the Local Binary Pattern (LBP) histogram of the current feature frame and the averaged feature frame. The frames which yield score greater than a predetermined threshold were regarded as frames with micro-expression.

A similar approach was carried out by [37], except that: (1) a denoising method was added before extracting the features, and; (2) the Histogram of Gradient was used instead of LBP. However, the database they tested on was not publicly available. Since the benchmark video sequences used in this paper [37] and that in [36] are different, their performances cannot be compared directly. Both papers claimed that the eye blinking movement is one type of the micro-expression. However, it was not detailed in the ground-truth and hence the frames containing eye blinking movements were annotated manually. A recent work by Wang et al. [38] proposed main directional maximal difference analysis for spotting facial movements from long-term videos.

To the best of our knowledge, there is only one recent work that attempted to combine both spotting and recognition of micro-expressions, which is the work of $\mathrm{Li}$ et al. [39]. They extended the work by Moilanen et al. [36], where after the spotting stage, the spotted microexpression frames (i.e., those with the onset and offset information) were concatenated to a single sequence for expression recognition. In the recognition task, they employed motion magnification technique and proposed a new feature extractor - the Histograms of Image Gradient Orientation. However, the recognition performance was poor compared to the state-of-the-art. Besides, the frame rate of the database is $25 \mathrm{fps}$, which means that the maximum frame number in a raw video sequence is only $1 / 5 s \times 25 \mathrm{fps}=5$.

\subsection{Apex spotting}

Apart from the aforementioned micro-expression frames searching approaches, the other technique used is to automatically spot the instance of the single apex frame in a video. The micro-expression information retrieved from that apex frame is expected to be insightful in both psychological and computer vision research purposes, because it contains the maximum facial muscle movements throughout the video sequence. Yan et al. [40] published the first work in spotting the apex frame. They employed two feature extractors (i.e., LBP and Constraint Local Models) and reported the average frame distance between the spotted apex and the ground-truth apex. The frame that has the highest feature difference between the first frame and the subsequent frames is defined to be the apex. However, there are two flaws in this work: (1) The average frame distance calculated was not in absolute mean, which led to incorrect results; (2) The method was validated by using only $\sim 20 \%$ of the video samples in the database (i.e., CASME II), hence not conclusive and convincing.

The second work on apex frame spotting was presented by Liong et al. [41], which differs from the first work by Yan et al. [40] as follows: (1) A divide-and-conquer strategy was implemented to locate the frame index of the apex, because the maximum difference between the first and the subsequent frames might not necessarily be the apex frame; (2) An extra feature extractor was added to confirm the reliability of the method proposed; (3) Selected important facial regions were considered for feature encoding instead of the whole face, and; (4) All the video sequences in the database (i.e., CASME II) were used for evaluation and the average frame distance between the spotted and groundtruth apex were computed in absolute mean.

Later, Liong et al. [42] spotted the micro-expression on long videos (i.e., SMIC-E-HS and CASME II-RAW databases). Specifically, long video refers to the raw video sequence which may include the frames with microexpressions as well as irrelevant motion that are present before the onset and after the offset. On the other hand, short video is a sub-sequence of the long video starting from the onset and ending with the offset. In other words, all frames before the onset frame and after the offset frame are excluded. A novel eye masking approach was also proposed to mitigate the issue where frames in the long videos may contain large and irrelevant movements such as eye blinking actions, which can potentially cause erroneous spotting. 


\section{4. "Less" is More?}

Considering these developments, we pose the following intriguing question: With the high-speed video capture of micro-expressions (100-200 fps), are all frames necessary to provide a sufficiently meaningful representation? While the works of Li et al. [14] and Le Ngo et al. [29, 43] showed that a reduced-size sequence can somewhat help retain the vital information necessary for a good representation, there are no existing investigations into the use of the apex frame. How meaningful is the so-called apex frame? Ekman [44] asserted that a "snapshot taken at an point when the expression is at its apex can easily convey the emotion message". A similar observation by Esposito [45] earmarked the apex as "the instant at which the indicators of emotion are most marked". Hence we can hypothesize that the apex frame offers the strongest signal that depicts the "momentary configuration" [44] of facial contraction.

In this paper, we propose a novel approach to microexpression recognition, where for each video sequence, we encode features from the representative apex frame with the onset frame as the reference frame. The onset frame is assumed to be the neutral face and is provided in all micro-expression databases (e.g., CAS(ME) ${ }^{2}$, CASME II and SMIC) while the apex frame labels are only available in $\mathrm{CAS}(\mathrm{ME})^{2}$ and CASME II. To solve the lack of apex information in SMIC, a binary search strategy was employed to spot the apex frame [41]. We renamed binary search to divide-and-conquer for a more general terminology to this scheme. Additionally, we introduce a new feature extractor called Bi-Weighted Oriented Optical Flow (Bi-WOOF), which is capable of representing the apex frame in a discriminative manner, emphasizing facial motion information at both bin and block levels. The histogram of optical flow orientations is weighted twice at different representation scales, namely, bins by the magnitudes of optical flow, and block regions by the magnitudes of optical strain. We establish our proposition by proving empirically through a comprehensive evaluation that was carried out on four notable databases.

The rest of this paper is organized as follows. Section 3 explains the proposed algorithm in detail. The descriptions of the databases used are discussed in Section 4, followed by Section 5 that reports the experiment results and discussion for the recognition of micro-expressions. Finally, conclusion is drawn in Section 6 .

\section{Proposed Algorithm}

The proposed micro-expression recognition system comprises of two components, namely, apex frame spotting, and micro-expression recognition. The architecture overview of the system is illustrated in Figure 3. The following subsections detail the steps involved.

\subsection{Apex Spotting}

To spot the apex frame, we employ the approach proposed by Liong et al. [41], which consists of five steps: (1) The facial landmark points are first annotated by using a landmark detector called Discriminative Response Map Fitting (DRMF) [46]; (2) The regions of interest that indicate the facial region with important micro-expression details are extracted according to the landmark coordinates; (3) The LBP feature descriptor is utilized to obtain the features of each frame in the video sequence (i.e., from onset to offset); (4) The feature difference between the onset and the rest of the frames are computed using the correlation coefficient formula, and finally; (5) A peak detector with divide-and-conquer strategy is utilized to search for the apex frame based on the LBP feature difference. Specifically, the procedures of divide-and-conquer methodology are: (A) The frame index of the peaks/ local maximum in the video sequence are detected by using a peak detector. (B) The frame sequence is divided into two equal halves (e.g., a 40 frames video sequence is split into two sub-sequences containing frame 1-20 and 21-40). (C) Magnitudes of the detected peaks are summed up for each of the sub-sequence. (D) The sub-sequence with the higher magnitude will be considered for the next computation step while the other sub-sequence will be discarded. (E) Steps (B) to (D) are repeated until the final peak (also known as apex frame) is found. Liong et al. [41] reported that the average estimated apex frame is 13 frames away from the ground-truths apex frames for divide-and-conquer methodology. Note that the micro-expression video has an average length of 68 frames. Figure 4 illustrates the apex frame spotting approach in a sample video. It can be seen that, the ground-truth apex (frame \#63) and the spotted apex (frame \#64) differ only by one frame.

\subsection{Micro-expression Recognition}

Here, we discuss a new feature descriptor, Bi-Weighted Oriented Optical Flow (Bi-WOOF) that represents a sequence of subtle expressions by using only two frames. As illustrated in Figure 5, the recognition algorithm contains three main steps: (1) The horizontal and vertical optical flow vectors between the apex and neutral frames are estimated; (2) The orientation, magnitude and optical strain of each pixel's location are computed from the respective two optical flow components; (3) A Bi-WOOF histogram is formed based on the orientation, with magnitude locally weighted and optical strain globally weighted.

\subsubsection{Optical flow estimation [47]}

Optical flow approximates the changes of an object's position between two frames that are sampled at slightly different times. It encodes the motion of an object in vector notation, which indicates the direction and intensity of the flow of each image pixel. The horizontal and vertical components of the optical flow are defined as: 


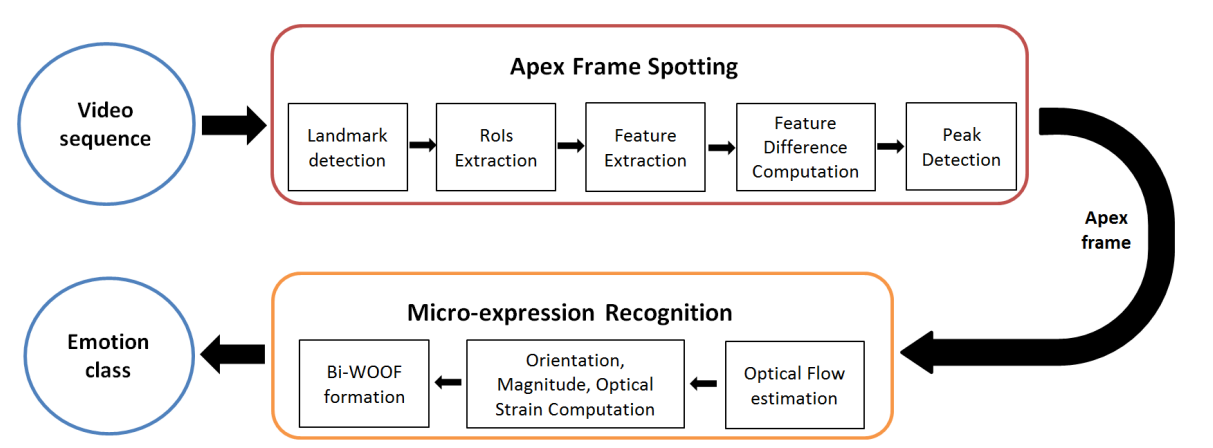

Figure 3: Framework of the proposed micro-expression recognition system

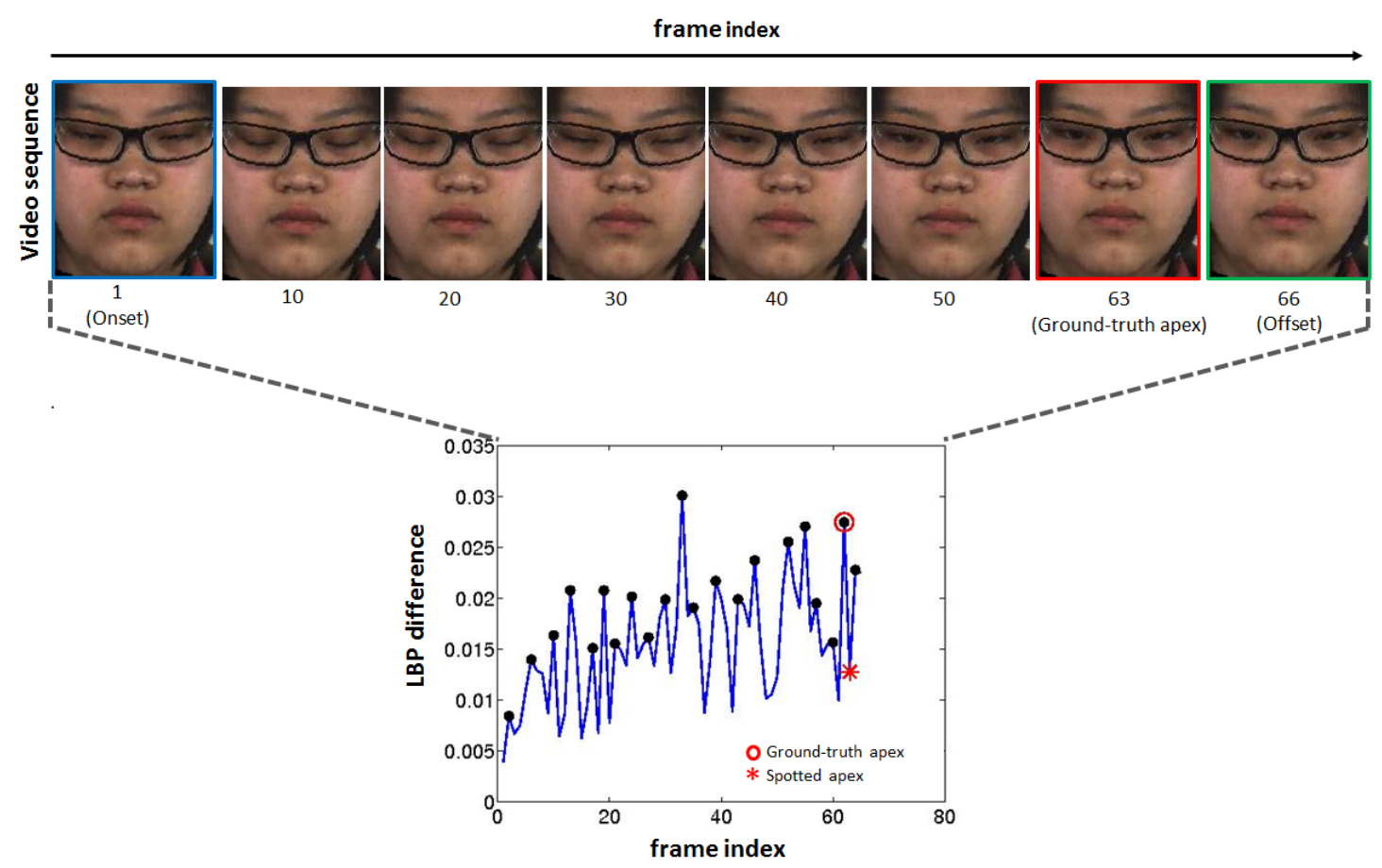

Figure 4: Illustration of the apex spotting in the video sequence (i.e., sub20-EP12_01 in CASME II [9] database) using LBP feature extractor with divide-and-conquer [41] strategy

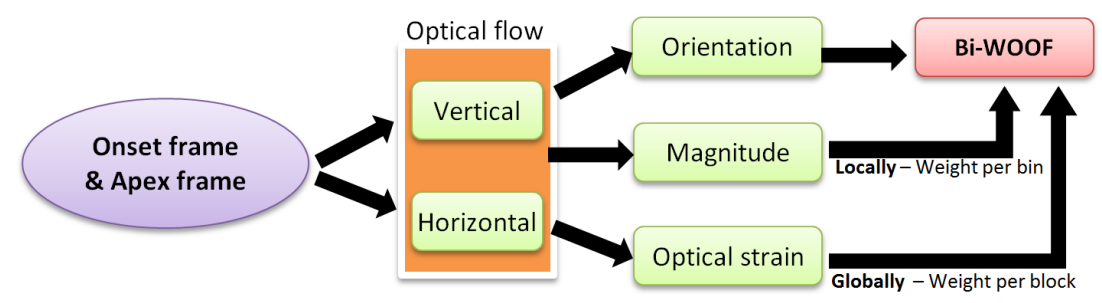

Figure 5: Flow diagram of micro-expression recognition system

$$
\vec{p}=\left[p=\frac{d x}{d t}, q=\frac{d y}{d t}\right]^{T}
$$

where $(d x, d y)$ indicate the changes along the horizontal and vertical dimensions, and $d t$ is the change in time. The optical flow constraint equation is given by:

$$
\nabla I \bullet \vec{p}+I_{t}=0
$$

where $\nabla I=\left(I_{x}, I_{y}\right)$ is the gradient vector of image intensity evaluated at $(x, y)$ and $I_{t}$ is the temporal gradient of the intensity functions.

We employed TV-L1 [48] for optical flow approximation due to its two major advantages, namely, better noise robustness and the ability to preserve flow discontinuities.

We first introduce and describe the notations which are used in the subsequent sections. A micro-expression video 
clip is denoted as:

$$
s_{i}=\left\{f_{i, j} \mid i=1, \ldots, n ; j=1, \ldots, F_{i}\right\},
$$

where $F_{i}$ is the total number of frames in the $i$-th sequence, which is taken from a collection of $n$ video sequences. For each video sequence, there is only one apex frame, $f_{i, a} \in$ $f_{i, 1}, \ldots, f_{i, F i}$, and it can be located at any frame index.

The optical flow vectors of the onset (assumed as neutral expression) and the apex frames are predicted then denoted by $f_{i, 1}$ and $f_{i, a}$, respectively. Hence, each video of resolution $X \times Y$ produces only one set of optical flow map, expressed as:

$$
\nu_{i}=\left\{\left(u_{x, y}, v_{x, y}\right) \mid x=1, \ldots, X ; y=1, \ldots, Y\right\}
$$

for $i \in 1,2, \ldots n$. Here, $\left(u_{x, y}, v_{x, y}\right)$ are the displacement vectors in the horizontal and vertical directions respectively.

\subsubsection{Computation of orientation, magnitude and optical strain}

Given the optical flow vectors, we derive three characteristics to describe the facial motion patterns: (1) magnitude: intensity of the pixel's movement; (2) orientation: direction of the flow motion, and; (3) optical strain: subtle deformation intensity.

In order to obtain the magnitude and orientation, the flow vectors, $\vec{o}=(p, q)$, are converted from euclidean coordinates to polar coordinates:

$$
\rho_{x, y}=\sqrt{p_{x, y^{2}}+q_{x, y}^{2}},
$$

and

$$
\theta_{x, y}=\tan ^{-1} \frac{q_{x, y}}{p_{x, y}},
$$

where $\rho$ and $\theta$ are the magnitude and orientation, respectively.

The next step is to compute the optical strain, $\varepsilon$, based on the optical flow vectors. For a sufficiently small facial pixel's movement, it is able to approximate the deformation intensity, also known as the infinitesimal strain tensor. In brief, the infinitesimal strain tensor is derived from the Lagrangian and Eulerian strain tensor after performing a geometric linearisation [49]. In terms of displacements, the typical infinitesimal strain $(\varepsilon)$ is defined as:

$$
\varepsilon=\frac{1}{2}\left[\nabla \mathbf{u}+(\nabla \mathbf{u})^{T}\right]
$$

where $\mathbf{u}=[u, v]^{T}$ is the displacement vector. It can also be re-written as:

$$
\varepsilon=\left[\begin{array}{cc}
\varepsilon_{x x}=\frac{\partial u}{\partial x} & \varepsilon_{x y}=\frac{1}{2}\left(\frac{\partial u}{\partial y}+\frac{\partial v}{\partial x}\right) \\
\varepsilon_{y x}=\frac{1}{2}\left(\frac{\partial v}{\partial x}+\frac{\partial u}{\partial y}\right) & \varepsilon_{y y}=\frac{\partial v}{\partial y}
\end{array}\right],
$$

where the diagonal strain components, $\left(\varepsilon_{x x}, \varepsilon_{y y}\right)$, are normal strain components and $\left(\varepsilon_{x y}, \varepsilon_{y x}\right)$ are shear strain components. Specifically, normal strain measures the changes in length along a specific direction, whereas shear strains measure the changes in two angular.

The optical strain magnitude for each pixel can be calculated by taking the sum of squares of the normal and shear strain components, expressed below:

$$
\begin{aligned}
\left|\varepsilon_{x, y}\right| & =\sqrt{\varepsilon_{x x^{2}}+\varepsilon_{y y^{2}}+\varepsilon_{x y}^{2}+\varepsilon_{y x^{2}}} \\
& =\sqrt{\frac{\partial u^{2}}{\partial x}+\frac{\partial v^{2}}{\partial y}+\frac{1}{2}\left(\frac{\partial u}{\partial x}+\frac{\partial u}{\partial x}\right)^{2}}
\end{aligned}
$$

\subsubsection{Bi-Weighted Oriented Optical Flow}

In this stage, we utilize the three aforementioned characteristics (i.e., orientation, magnitude and optical strain images for every video) to build a block-based Bi-Weighted Oriented Optical Flow.

The three characteristic images are partitioned equally into $N \times N$ non-overlapping blocks. For each block, the orientations $\theta_{x, y} \in[-\pi, \pi]$ are binned and locally weighted according to its magnitude $\rho_{x, y}$. Thus, the range of each histogram bin is:

$$
-\pi+\frac{2 \pi c}{C} \leq \theta_{x, y}<-\pi+\frac{2 \pi(c+1)}{C},
$$

where bin $c \in\{1,2, \ldots, C\}$, and $C$ denotes the total number of histogram bins.

To obtain the global weight $\zeta_{b_{1}, b_{2}}$ for each block, we utilize the optical strain magnitude $\varepsilon_{x, y}$ as follows:

$$
\zeta_{b_{1}, b_{2}}=\frac{1}{H L} \sum_{y=\left(b_{2}-1\right) H+1}^{b_{2} H} \sum_{x=\left(b_{1}-1\right) L+1}^{b_{1} L} \varepsilon_{x, y},
$$

where $L=\frac{X}{N}, H=\frac{Y}{N}$, the $b_{1}$ and $b_{2}$ are the block indices such that $b_{1}, b_{2} \in 1,2, \ldots, N, X \times Y$ is the dimensions (viz., width-by-height) of the video frame.

Lastly, the coefficients of $\zeta_{b_{1}, b_{2}}$ are multiplied with the locally weighted histogram bins to their corresponding blocks. The histogram bins of each block are concatenated to form the resultant feature histogram.

In contrast to the conventional Histogram of Oriented Optical Flow (HOOF) [50], our proposed orientation histogram bins have equal votes. Here, we consider both the magnitude and optical strain values as the weighting schemes to highlight the importance of each optical flow. Hence, a larger intensity of the pixel's movement or deformation contributes more effect to the histogram, whereas noisy optical flows with small intensities reduce the significance of the features.

The overall process flow of obtaining the locally and globally weighted features is illustrated in Figure 6 .

\section{Experiment}

\subsection{Datasets}

To evaluate the performance of the proposed algorithm, the experiments were carried out on five recent spontaneous micro-expression databases, namely $\mathrm{CAS}(\mathrm{ME})^{2}$ [16], 


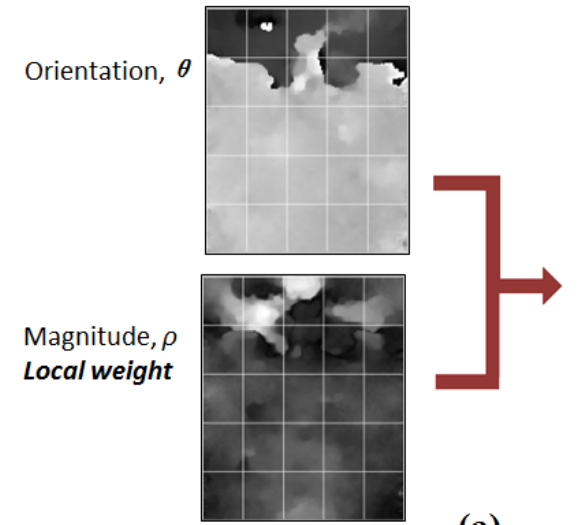

(a)
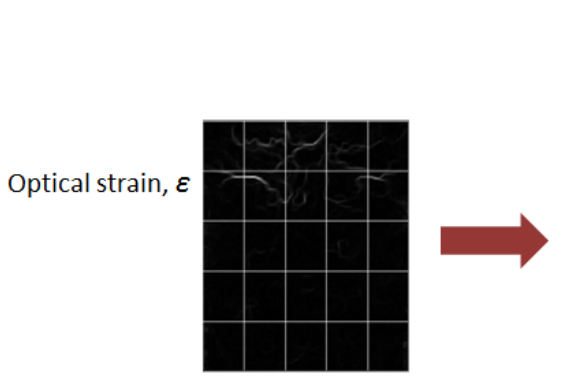

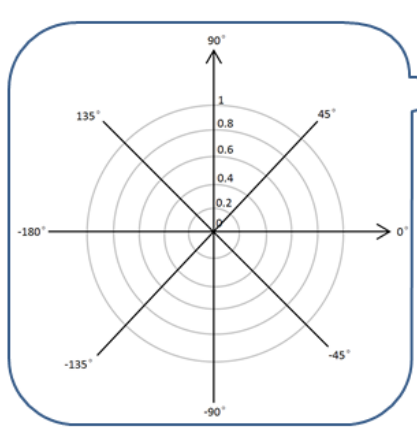

(c)

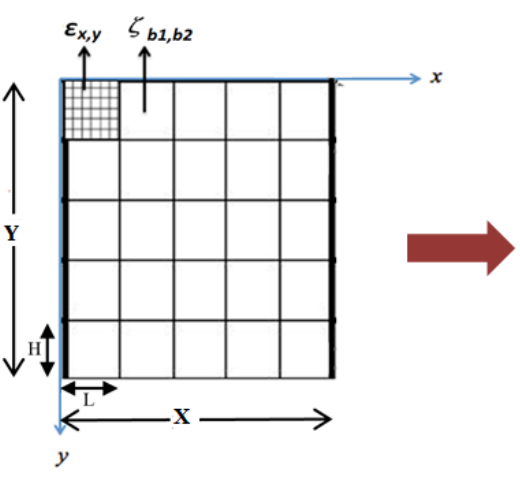

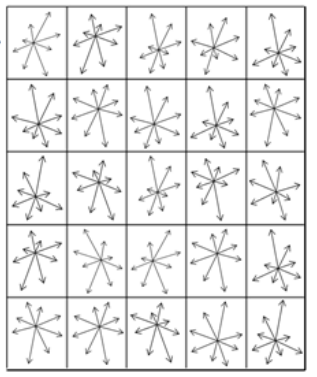

Locally weighted HOOF

(b)

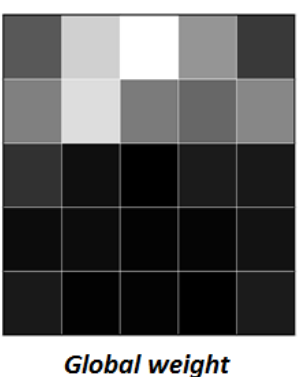

(d)

Figure 6: The process of Bi-WOOF feature extraction for a video sample: (a) $\theta$ and $\rho$ images are divided into $N \times N$ blocks. In each block, the values of $\rho$ for each pixel are treated as local weights to multiply with their respective $\theta$ histogram bins; (b) It forms a locally weighted HOOF with feature size of $N \times N \times C$; (c) $\zeta_{b 1, b 2}$ denotes the global weighting matrix, which is derived from $\varepsilon$ image; (d) Finally, $\zeta_{b 1, b 2}$ are multiplied with their corresponding locally weighted HOOF.

CASME II [9], SMIC-HS [14], SMIC-VIS [14] and SMICNIR [14]. Note that all these databases are recorded in a constrained laboratory condition due to the subtlety of micro-expressions.

\subsubsection{CASME II}

CASME II consists of five classes of expressions: surprise (25 samples), repression (27 samples), happiness (23 samples), disgust (63 samples) and others (99 samples). Each video clip contains only one micro-expression. Thus, there is a total of 246 video sequences. The emotion labels were marked by two coders with the reliability of 0.85 . The expressions were elicited from 26 subjects with the mean age of 22 years old, and recorded using the camera - Point Grey GRAS-03K2C. The video resolution and frame rate of the camera are $640 \times 480$ pixels and 200 fps respectively. This database provides the cropped video sequences, where only the face region is shown while the unnecessary background has been eliminated. The cropped images have an average spatial resolution of $170 \times 140$ pixels, and each video consists of 68 frames (viz., 0.34s). The video with the highest and lowest number of frames are 141 (viz., $0.71 s$ ) and 24 (viz., $0.12 s$ ), respectively. The frame index (i.e., frame number) for onset, apex and offset of each video sequence are provided. To perform the recognition task on this micro-expression dataset, the block-based LBP-TOP feature was considered. The features were then classified by a Support Vector Machine (SVM) with leave-one-videoout cross-validation (LOVOCV) protocol.

\subsection{2. $S M I C$}

SMIC includes three sub-datasets, which are SMIC-HS, SMIC-VIS and SMIC-NIR. The data composition of these datasets are detailed in Table 1 . It is noteworthy that all eight participants who appeared in the VIS and NIR datasets were also involved in HS dataset elicitation. During the recording process, three cameras (i.e., HS, VIS and NIR) were recording simultaneously. The cameras were placed parallel to each other at the middle-top of the monitor. The groundtruth of the frame indices of onset and offset for each video clip in SMIC are given, but not the apex frame. The three-class recognition task was carried out for the three SMIC datasets individually by utilizing block-based LBP-TOP as the feature extractor and SVMLOSOCV (leave-one-subject-out cross-validation) as the classifier.

\subsection{3. $C A S(M E)^{2}$}

$\mathrm{CAS}(\mathrm{ME})^{2}$ dataset has two major parts (A and B). Part A consists of 87 long videos, containing both spontaneous macro-expressions and micro-expressions. Part 
B contains 300 short videos (i.e., cropped faces) spontaneous macro-expression samples and 57 micro-expression samples. To evaluate the proposed method, we only consider the cropped micro-expression videos (i.e., 57 samples in total). However, we discovered three samples are missing from the dataset provided. Hence, 54 microexpression video clips are used in the experiment. The micro-expression video sequences are elicited from 14 participants. This dataset provides the cropped face video sequence. The videos are recorded using Logitech Pro C920 camera with a temporal resolution of $30 \mathrm{fps}$ and spatial resolution of $640 \times 480$ pixels. It composes of four classes of expressions: negative (21 samples), others (19 samples), surprise ( 8 samples) and positive (6 samples). We resized the images to $170 \times 140$ pixels for experiment purpose. The average number of frames of the microexpression video sequences is 6 frames (viz., $0.2 s$ ). The video with the highest and lowest number of frames are 10 (viz., 0.33s) and 4 (viz., 0.13s), respectively. The groundtruth frame indices for onset, apex and offset of each video sequence are also provided. To annotate the emotion label for each video sequence, a combination of AUs, emotion types of expression-elicitation video and self-reported are considered. The highest accuracy for the four-class recognition task reported in the original paper [16] is $40.95 \%$. It is obtained by adopting LBP-TOP feature extractor and SVM-LOSOCV classifier.

\subsubsection{Experiment Settings}

The aforementioned databases (i.e., $\mathrm{CAS}(\mathrm{ME})^{2}$, CASME II and SMIC) have imbalance distribution of the emotion types. Therefore, it is necessary to measure the recognition performance of the proposed method using F-measure, which was also suggested in [51]. Specifically, F-measure is defined as:

$$
\text { F-measure }:=2 \times \frac{\text { Precision } \times \text { Recall }}{\text { Precision }+ \text { Recall }}
$$

for

$$
\text { Recall }:=\frac{\sum_{i=1}^{M} \mathrm{TP}_{i}}{\sum_{i=1}^{M} \mathrm{TP}_{i}+\sum_{i=1}^{M} \mathrm{FN}_{i}}
$$

and

$$
\text { Precision }:=\frac{\sum_{i=1}^{M} \mathrm{TP}_{i}}{\sum_{i=1}^{M} \mathrm{TP}_{i}+\sum_{i=1}^{M} \mathrm{FP}_{i}}
$$

where $M$ is the number of classes; TP, FN and FP are the true positive, false negative and false positive, respectively.

On the other hand, to avoid person dependent issue in the classification process, we employed LOSOCV strategy in the linear SVM classifier setting. In LOSOCV, the features of the sample videos in one subject are treated as the testing data and the remaining features from rest of the subjects become the training data. Then, this process is repeated for $k$ times, where $k$ is the number of subjects in the database. Finally, the recognition results for all the subjects are averaged to compute the recognition rate.
For the block-based feature extraction methods (i.e., LBP, LBP-TOP and proposed algorithm), we standardized the block sizes to $5 \times 5$ and $8 \times 8$ for the SMIC and CASME II datasets, respectively, as we discovered that these block settings generated reasonably good recognition performance in all cases. Since CAS(ME) ${ }^{2}$ was only made public recently, there is still no method designed and tested on this dataset in the literature. Hence, we report the recognition results for various block sizes using the baseline LBP-TOP and our proposed Bi-WOOF methods.

\section{Results and Discussion}

In this section, we present the recognition results with detailed analysis and benchmarking against state-of-theart methods. We also examine the computational efficiency of our proposed method, and lay down some key propositions derived from observations in this work.

\subsection{Recognition Results}

We report the results in two parts, according to the databases: (i) CAS(ME) ${ }^{2}$ (in Table 2) and (ii) CASME II, SMIC-HS, SMIC-VIS and SMIC-NIR (in Table 3).

Table 2 records the recognition performance on $\mathrm{CAS}(\mathrm{ME})^{2}$ with various block sizes by employing the baseline LBP-TOP and our proposed Bi-WOOF feature extractors. This is because the original paper [16] did not perform recognition task solely on the micro-expression samples, instead the result reported was tested on the mixed macro-expression and micro-expression samples. We record both the F-measure and Accuracy measurements for different blocks sizes, including $5 \times 5,6 \times 6$, $7 \times 7$ and $8 \times 8$ for both feature extraction methods. The best F-measure performance achieved by LBP-TOP is $41 \%$, while Bi-WOOF method achieves $47 \%$. Both results are obtained when block size is set to $6 \times 6$.

The micro-expression recognition performances of the proposed method (i.e., Bi-WOOF) and the other conventional feature extraction methods evaluated on CASME II, SMIC-HS, SMIC-VIS and SMIC-NIR databases are shown in Table 3. Note that the sequence-based methods \#1 to \#13 considered all frames in the video sequence (i.e., frames from onset to offset). Meanwhile, methods \#14 to \#19 consider only information from the apex and onset frames, whereby only two images are processed to extract features. We refer to these as apex-based methods.

Essentially, our proposed apex-based approach requires determining the apex frame for each video sequence. Although the SMIC datasets (i.e., HS, VIS and NIR) did not provide the ground-truth apex frame indices, we utilize the divide-and-conquer strategy proposed in [41] to spot the apex frame. For CASME II, the ground-truth apex frame indices are already provided, so we can use them directly. 
Table 1: Detailed information of the SMIC-HS, SMIC-VIS and SMIC-HR datasets

\begin{tabular}{|c|c|c|c|c|}
\hline Datasets & & SMIC-HS & SMIC-VIS & SMIC-NIR \\
\hline Participants & & 16 & 8 & 8 \\
\hline \multirow[t]{2}{*}{ Camera } & Type & $\begin{array}{l}\text { PixeLINK } \\
\text { PL-B774U }\end{array}$ & $\begin{array}{l}\text { Visual } \\
\text { camera }\end{array}$ & $\begin{array}{c}\text { Near-infrared } \\
\text { camera }\end{array}$ \\
\hline & Frame rate (fps) & 100 & 25 & 25 \\
\hline \multirow{4}{*}{ Expression } & Positive & 51 & 28 & 28 \\
\hline & Negative & 70 & 23 & 23 \\
\hline & Surprise & 43 & 20 & 20 \\
\hline & Total & 164 & 71 & 71 \\
\hline \multirow{2}{*}{$\begin{array}{l}\text { Image } \\
\text { resolution }\end{array}$} & Raw & $640 \times 480$ & $640 \times 480$ & $640 \times 480$ \\
\hline & Cropped (avg.) & $170 \times 140$ & $170 \times 140$ & $170 \times 140$ \\
\hline \multirow{3}{*}{$\begin{array}{l}\text { Frame } \\
\text { number }\end{array}$} & Average & 34 & 10 & 10 \\
\hline & Maximum & 58 & 13 & 13 \\
\hline & Minimum & 11 & 4 & 4 \\
\hline \multirow{3}{*}{$\begin{array}{c}\text { Video } \\
\text { duration }(s)\end{array}$} & Average & 0.34 & 0.4 & 0.4 \\
\hline & Maximum & 0.58 & 0.52 & 0.52 \\
\hline & Minimum & 0.11 & 0.16 & 0.16 \\
\hline
\end{tabular}

Table 2: Micro-expression recognition results (\%) on $\mathrm{CAS}(\mathrm{ME})^{2}$ with different number of block size for the LBP-TOP and Bi-WOOF feature extractors

\begin{tabular}{lcccc} 
& \multicolumn{2}{c}{ F-measure } & \multicolumn{2}{c}{ Accuracy } \\
\hline Block & LBP-TOP & Bi-WOOF & LBP-TOP & Bi-WOOF \\
\hline $5 \times 5$ & .28 & $\mathbf{. 4 7}$ & 46.30 & $\mathbf{5 9 . 2 6}$ \\
$6 \times 6$ & .41 & $\mathbf{. 4 7}$ & 48.15 & $\mathbf{5 9 . 2 6}$ \\
$7 \times 7$ & .26 & $\mathbf{. 4 6}$ & 44.44 & $\mathbf{5 9 . 2 6}$ \\
$8 \times 8$ & .28 & $\mathbf{. 4 7}$ & 48.15 & $\mathbf{5 9 . 2 6}$ \\
\hline
\end{tabular}

In order to validate the importance of the apex frame, we also randomly select one frame from each video sequence. Features are then computed using the apex/ random frame and the onset (reference) frame using LBP , HOOF and Bi-WOOF descriptors. The recognition performances of the random frame selection approaches (repeated for 10 times) are reported as methods \#14, \#16 and \#18 while the apex-frame approaches are reported as methods \#15, \#17 and \#19. We observe that the utilization of the apex frame always yields better recognition results when compared to using random frames. As such, it can be concluded that the apex frame plays an important role in forming discriminative features.

For method \#1 (i.e., LBP-TOP), also referred to as the baseline, we reproduced the experiments for the four datasets based on the original papers $[9,14]$. The recognition rates for methods \#2 to \#11 are reported from their respective works of the same experimental protocol. Besides, we replicated method \#12 and evaluate it on CASME II database. This is because the original paper [28] classifies the emotion into 4 types (i.e., positive, negative, surprise and others). For a fair comparison with our proposed method, we re-categorize the emotions into 5 types (i.e., happiness, disgust, repression, surprise and others). For method \#13, Bi-WOOF is applied on all frames in the video sequence. The features were computed by first estimating the three characteristics of the optical flow (i.e., orientation, magnitude and strain) between the onset and each subsequent frame (i.e., $\left\{f_{i, 1}, f_{i, j}\right\}, j \in 2, \ldots, F_{i}$ ). Next, Bi-WOOF was computed for each pair of frames to obtain the resultant histogram.

LBP was applied on the difference image to compute the features in methods \#14 and \#15. Note that the image subtraction process is only applicable for methods \#14 (LBP - random \& onset) and \#15 (LBP - apex \& onset). This is because LBP feature extractor can only capture the spatial features of an image and it is incapable of extracting the temporal features of two images. Specifically, the spatial features extracted from the apex frame and the onset frame are not correlated. Hence, we perform an image subtraction process in order to generate a single image from two images (i.e., apex / random frame and onset frame). This image subtraction process can remove a person's identity while preserving the characteristics of facial micro-movements. Besides, for the apex-based approaches, we also evaluated the HOOF feature (i.e., methods \#16 and \#17) by binning the optical flow orientation, which is computed between the apex / random frame and the onset frame, to form the feature histogram.

Table 3 suggests that the proposed algorithm (viz., \#19) achieves promising results in all four datasets. More precisely, it outperformed all the other methods in CASME II. In addition, for SMIC-VIS and SMIC-NIR, the results of the proposed method are comparable to those of \#9, viz., FDM method. 
Table 3: Comparison of micro-expression recognition performance in terms of F-measure on the CASME II, SMIC-HS, SMIC-VIS and SMIC-NIR databases for the state-of-the-art feature extraction methods, and the proposed apex frame methods

\begin{tabular}{|c|c|c|c|c|c|c|}
\hline & & Methods & CASME II & SMIC-HS & SMIC-VIS & SMIC-NIR \\
\hline \multirow{13}{*}{ 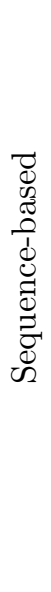 } & 1 & LBP-TOP $[9,14]$ & .39 & .39 & .39 & .40 \\
\hline & 2 & OSF $[24]$ & - & .45 & - & - \\
\hline & 3 & $\operatorname{STM}[51]$ & .33 & .47 & - & - \\
\hline & 4 & OSW $[25]$ & .38 & .54 & - & - \\
\hline & 5 & LBP-SIP [21] & .40 & .55 & - & - \\
\hline & 6 & $\mathrm{MRW}[26]$ & .43 & .35 & - & - \\
\hline & 7 & STLBP-IP [22] & .57 & .58 & - & - \\
\hline & 8 & $\mathrm{OSF}+\mathrm{OSW}[52]$ & .29 & .53 & - & - \\
\hline & 9 & FDM $[30]$ & .30 & .54 & .60 & .60 \\
\hline & 10 & $\begin{array}{l}\text { Sparse } \\
\text { Sampling [29] }\end{array}$ & .51 & .60 & - & - \\
\hline & 11 & STCLQP [23] & .58 & .64 & - & - \\
\hline & 12 & MDMO [28] & .44 & - & - & - \\
\hline & 13 & $\mathrm{Bi}-\mathrm{WOOF}$ & .56 & .53 & .62 & .57 \\
\hline \multirow{6}{*}{ 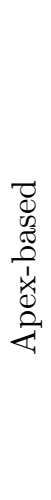 } & 14 & $\begin{array}{l}\text { LBP } \\
\text { (random \& onset) }\end{array}$ & .38 & .40 & .48 & .51 \\
\hline & 15 & $\begin{array}{l}\text { LBP } \\
\text { (apex \& onset) }\end{array}$ & .41 & .45 & .49 & .54 \\
\hline & 16 & $\begin{array}{l}\text { HOOF } \\
\text { (random \& onset) }\end{array}$ & .41 & .40 & .51 & .50 \\
\hline & 17 & $\begin{array}{l}\text { HOOF } \\
\text { (apex \& onset) }\end{array}$ & .43 & .48 & .49 & .47 \\
\hline & 18 & $\begin{array}{l}\text { Bi-WOOF } \\
\text { (random \& onset) }\end{array}$ & .50 & .46 & .56 & .50 \\
\hline & 19 & $\begin{array}{l}\text { Bi-WOOF } \\
\text { (apex \& onset) }\end{array}$ & .61 & .62 & .58 & .58 \\
\hline
\end{tabular}

\subsection{Analysis and Discussion}

To further analyze the recognition performances, we provide the confusion matrices for the selected databases. Firstly, for $\mathrm{CAS}(\mathrm{ME})^{2}$, as tabulated in Table 4, it can be seen that the recognition rate using Bi-WOOF method outperforms LBP-TOP method for all block sizes. Therefore, it can be concluded that the Bi-WOOF method is superior compared to the baseline method.

On the other hand, for the CASME II and SMIC databases, we only present the confusion matrices for the high frame rate databases, namely, CASME II and SMICHS. This is because most works in the literature tested on these two spontaneous micro-expression databases, making performance comparisons possible. It is worth highlighting that a number of works in literature such as [27, 28], perform classification of micro-expressions in CASME II based on four categories (i.e., negative, positive, surprise and others), instead of the usual five (i.e., disgust, happiness, tense, surprise and repression) as used in most works.

The confusion matrices are recorded in Tables 5 and 6 for CASME II and SMIC-HS, respectively. It is observed that there are significant improvements in classification performance for all kinds of expression when employing Bi-WOOF (apex \& onset) when compared to the baselines. More concretely, in CASME II, the recognition rate of surprise, disgust, repression, happiness and other expressions were improved by $44 \%, 30 \%, 22 \%, 13 \%$ and $4 \%$, respectively. Furthermore, for SMIC-HS, the recognition rate of the expressions of negative, surprise and positive were improved by $31 \%, 19 \%$ and $18 \%$, respectively.

Figure 7 exemplifies the components derived from optical flow using onset and apex frames of the video sample "s04_sur_01" in SMIC-HS, where the micro-expression of surprise is shown. Referring to the labeling criteria of the emotion in [9], the changes in facial muscles are centering at the eyebrow regions. We can hardly tell the facial movements in Figure 7a, 7b and 7c. For Figure $7 \mathrm{~d}$, a noticeable amount of the muscular changes are occurring at the upper part of the face, whereas in Figure 7e, the eyebrows regions have obvious facial movement. Since magnitude information emphasizes the amplitude of the facial changes, we exploit it as local weight. Due to the computation of higher order derivatives in obtaining the optical strain magnitudes, optical strain has the ability to remove the noise and preserve large motion changes. 


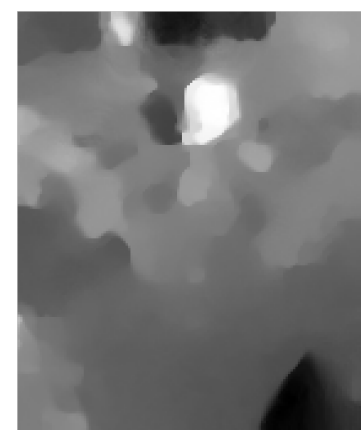

(a) $p$

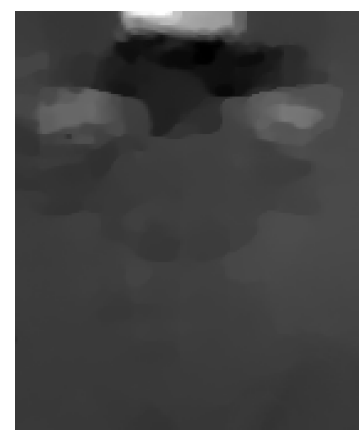

(b) $q$

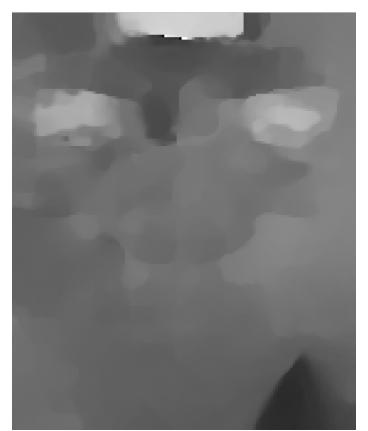

(c) $\theta$

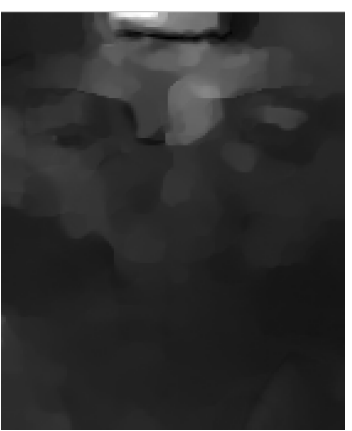

(d) $\rho$

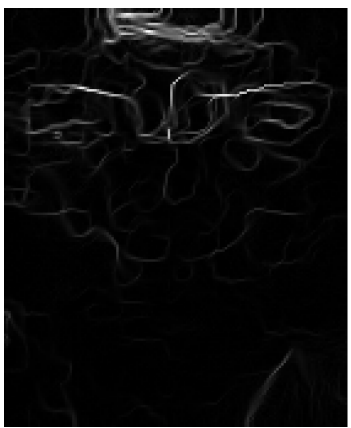

(e) $\varepsilon$

Figure 7: Illustration of components derived from optical flow using onset and apex frames of a video: (a) Horizontal vector of optical flow, $p$; (b) Vertical vector of optical flow, $q$; (c) Orientation, $\theta$; (d) Magnitude, $\rho$; (e) Optical strain, $\varepsilon$

Table 4: Confusion matrices of baseline and Bi-WOOF (apex \& onset) for the recognition task on $\mathrm{CAS}\left(\mathrm{ME}^{2}\right)$ database for block size of 6, where the emotion types are, POS: positive; NEG: negative; SUR: surprise; OTH: others

(a) Baseline

\begin{tabular}{lcccc} 
& POS & NEG & SUR & OTH \\
\hline POS & $\mathbf{. 1 7}$ & .33 & 0 & .50 \\
NEG & 0 & $\mathbf{. 6 7}$ & 0 & .33 \\
SUR & 0 & .38 & $\mathbf{0}$ & .63 \\
OTH & 0 & .42 & 0 & $\mathbf{. 5 8}$ \\
\hline
\end{tabular}

(b) Bi-WOOF (apex \& onset)

\begin{tabular}{lcccc} 
& POS & NEG & SUR & OTH \\
\hline POS & $\mathbf{0}$ & 0 & .50 & .50 \\
NEG & 0 &. $\mathbf{7 1}$ & .05 & .24 \\
SUR & .25 & .13 & $\mathbf{. 5 0}$ & .13 \\
OTH & .16 & .16 & 0 & $\mathbf{. 6 8}$ \\
\hline
\end{tabular}

We exploit these characteristics to build the global weight. In addition, [24] demonstrated that optical strain globally weighted on the LBP-TOP features produced better recognition results when compared to results obtained without the weighting.

Based on the results of F-measure and confusion matrices, it is observed that extracting the features of two images only (i.e., apex and onset frame) using the proposed method (i.e., Bi-WOOF) is able to yield superior recognition performance for the micro-expression databases considered, especially in CASME II and SMIC-HS, which have high temporal resolution (i.e., $\geq 100 \mathrm{fps}$ ).

The number of histogram bins $C$ in Eq. (10) is empirically determined to be 8 for both the CASME II and SMIC-HS databases. Table 7 quantitatively illustrates the relationship between the recognition performance and the histogram bins. It can be seen that with histogram bin $=$ 8, the Bi-WOOF feature extractor achieves the best recognition results on both CASME II and SMIC-HS databases.
Table 5: Confusion matrices of baseline and Bi-WOOF (apex \& onset) for the recognition task on CASME II database, where the emotion types are, DIS: disgust; HAP: happiness; OTH: others; SUR: surprise; and REP:repression

(a) Baseline

\begin{tabular}{lccccc} 
& DIS & HAP & OTH & SUR & REP \\
\hline DIS &. $\mathbf{2 0}$ & .11 & .66 & .02 & .02 \\
HAP & .09 &. $\mathbf{4 7}$ & .25 & 0 & .19 \\
OTH & .21 & .12 & $\mathbf{. 5 8}$ & .08 & 0 \\
SUR & .12 & .36 & .20 & $\mathbf{. 3 2}$ & 0 \\
REP & .07 & .33 & .26 & .04 & $\mathbf{. 3 0}$
\end{tabular}

(b) Bi-WOOF (apex \& onset)

\begin{tabular}{lccccc} 
& DIS & HAP & OTH & SUR & REP \\
\hline DIS & $\mathbf{. 4 9}$ & .07 & .44 & 0 & 0 \\
HAP & .03 & $\mathbf{. 5 9}$ & .28 & .03 & .06 \\
OTH & .21 & .09 & $\mathbf{. 6 2}$ & .01 & .06 \\
SUR & .04 & .12 & .08 & $\mathbf{. 7 6}$ & 0 \\
REP & .07 & .19 & .22 & 0 & $\mathbf{. 5 2}$ \\
\hline
\end{tabular}

We provide in Table 8 a closer look into the effects of applying (and not applying) the global and local weighting schemes on the Bi-WOOF features. Results on both SMIC-HS and CASME II are in agreement that the flow orientations are best weighted by their magnitudes, while the strain magnitudes are suitable as weights for the blocks. Results are the poorest when no global weighting is applied, which shows the importance of altering the prominence of features in different blocks.

\subsection{Computational Time}

We examine the computational efficiency of Bi-WOOF in SMIC-HS database on both the whole sequence and two images (i.e., apex and onset), which are the methods \#1 and \#15 in Table 3, respectively. The average duration taken per video for the execution of the micro-expression recognition system for the whole sequence and two images 
Table 6: Confusion matrices of baseline and Bi-WOOF (apex \& onset) for the recognition task on SMIC-HS database, where the emotion types are, NEG: negative; POS: positive; and SUR:surprise

(a) Baseline

\begin{tabular}{cccc} 
& NEG & POS & SUR \\
\hline NEG & $\mathbf{. 3 4}$ & .29 & .37 \\
POS & .41 & $\mathbf{. 3 9}$ & .20 \\
SUR & .37 & .19 & $\mathbf{. 4 4}$ \\
\hline
\end{tabular}

(b) Bi-WOOF (apex \& onset)

\begin{tabular}{cccc} 
& NEG & POS & SUR \\
\hline NEG & $\mathbf{. 6 6}$ & .23 & .11 \\
POS & .27 & $\mathbf{. 5 7}$ & .16 \\
SUR & .23 & .14 & $\mathbf{. 6 3}$ \\
\hline
\end{tabular}

Table 7: Micro-expression recognition results (\%) on SMIC-HS and CASME II databases with different number of histogram bins used for the Bi-WOOF feature extractor

\begin{tabular}{lcccc}
\cline { 2 - 5 } & \multicolumn{2}{c}{ CASME II } & \multicolumn{2}{c}{ SMIC-HS } \\
\hline Bin & F-measure & Accuracy & F-measure & Accuracy \\
\hline 1 & .39 & 46.09 & .46 & 45.12 \\
2 & .61 & 57.20 & .50 & 50.00 \\
3 & .59 & 55.56 & .49 & 48.78 \\
4 & .54 & 51.03 & .58 & 58.54 \\
5 & .60 & 58.02 & .53 & 54.27 \\
6 & .58 & 54.32 & .54 & 54.27 \\
7 & .57 & 54.32 & .50 & 50.00 \\
8 & .61 & $\mathbf{5 8 . 8 5}$ & $\mathbf{. 6 2}$ & $\mathbf{6 2 . 2 0}$ \\
9 & .59 & 56.38 & .49 & 49.39 \\
10 & .61 & 59.67 & .59 & 58.54 \\
\hline
\end{tabular}

in MATLAB implementation were $128.7134 \mathrm{~s}$ and $3.9499 \mathrm{~s}$ respectively. The time considered for this recognition system includes: (1) Spotting the apex frame using the divideand-conquer strategy; (2) Estimation of the horizontal and vertical components of optical flow; (3) Computation of orientation, magnitude and optical strain images; (4) Generation of Bi-WOOF histogram; (5) Expression classification in SVM. Both experiments were carried out on an Intel Core i7-4770 CPU $3.40 \mathrm{GHz}$ processor. Results suggest that the case of two images is $~ 33$ times faster than the case of whole sequence. It is indisputable that extracting the features from only two images is significantly faster than the whole sequence because lesser images are involved in the computation, and hence the volume of data to process is less.

\section{4. "Prima facie"}

At this juncture, we have established two strong propositions, which are by no means conclusive as further extensive research can provide further validation:
Table 8: Recognition performance (F-measure) with different combination of local and global weights used for Bi-WOOF

(a) SMIC-HS

\begin{tabular}{ccccc} 
& & \multicolumn{3}{c}{ Local } \\
\cline { 3 - 5 } & Weights & None & Flow & Strain \\
\hline $\overrightarrow{\widetilde{\sigma}}$ & None & .44 & .42 & .43 \\
$\overrightarrow{0}$ & Flow & .51 & .52 & .50 \\
$\overrightarrow{0}$ & Strain & .54 & $\mathbf{. 6 2}$ & .59 \\
\hline
\end{tabular}

(b) CASME II

\begin{tabular}{ccccc} 
& & \multicolumn{3}{c}{ Local } \\
\cline { 3 - 5 } & Weights & None & Flow & Strain \\
\hline $\overrightarrow{\widetilde{\sigma}}$ & None & .43 & .52 & .49 \\
$\overrightarrow{0}$ & Flow & .53 & .58 & .56 \\
$\overrightarrow{0}$ & Strain & .59 & $\mathbf{. 6 1}$ & .59 \\
\hline
\end{tabular}

1. The apex frame is the most important frame in a micro-expression clip, that it contains the most intense or expressive micro-expression information. Ekman's [44] and Esposito's [45] suggestions are validated by our use of the apex frame to characterize the change in facial contraction, a property best captured by the proposed Bi-WOOF descriptor which considers both facial flow and strain information. Control experiments using random frame selection (as the supposed apex frame) substantiates this fact. Perhaps, in future work, it will be interesting to know to what extent an imprecise apex frame (for instance, a detected apex frame that is located a few frames away) could influence the recognition performance. Also, further insights into locating the apices of specific facial Action Units (AUs) could possibly provide even better discrimination between types of micro-expressions.

2. The apex frame is sufficient for microexpression recognition. A majority of recent stateof-the-art methods promote the use of the entire video sequence, or a reduced set of frames [14, 29]. In this work, we advocate the opposite idea that, "less is more", supported by our hypothesis that a large number of frames does not guarantee a high recognition accuracy, particularly in the case when highspeed cameras are employed (e.g., for CASME II and SMIC-HS datasets). Comparisons against conventional sequence-based methods show that the use of the apex frame can provide more valuable information than a series of frames, what more at a much lower cost. At this juncture, it is premature to ascertain specific reasons behind this finding. Future directions point towards a detailed investigation into 
how and where micro-expression cues reside within the sequence itself.

\section{Conclusion}

In the recent few years, a number of research groups have attempted to improve the accuracy of micro-expression recognition by designing a variety of feature extractors that can best capture the subtle facial changes [21, 22, 28], while a few other works $[14,29,43]$ have sought out ways to reduce information redundancy in micro-expressions (using only a portion of all frames) before recognizing them.

In this paper, we demonstrated that it is sufficient to encode facial micro-expression features by utilizing only the apex frame (and onset frame as reference frame). To the best of our knowledge, this is the first attempt at recognizing micro-expressions in video using only the apex frame. For databases that do not provide apex frame annotations, the apex frame can be acquired by automatic spotting method based on a divide-and-conquer search strategy proposed in our recent work [41]. We also proposed a novel feature extractor, namely, Bi-Weighted Oriented Optical Flow (Bi-WOOF), which can concisely describe discriminately weighted motion features extracted from the apex and onset frames. As its name implies, the optical flow histogram features (bins) are locally weighted by their own magnitudes while facial regions (blocks) are globally weighted by the magnitude of optical strain - a reliable measure of subtle deformation.

Experiments conducted on five publicly available microexpression databases, namely, CAS $(\mathrm{ME})^{2}$, CASME II, SMIC-HS, SMIC-NIR and SMIC-VIS, demonstrated the effectiveness and efficiency of the proposed approach. Using a single apex frame for micro-expression recognition, the two high frame rate databases, i.e., CASME II and SMIC-HS, both achieved the promising recognition rate of $61 \%$ and $62 \%$, respectively, when compared to the stateof-the-art methods.

\section{Acknowledgments}

The authors would like to thank colleagues and anonymous reviewers for their valuable comments and suggestions to improve the quality of the paper. This work was supported in part by Telekom Malaysia R\&D Grant under Project 2beAware ((MMUE/140098), MOHE Grant FRGS/1/2016/ICT02/MMU/02/2, Multimedia University and University of Malaya.

\section{References}

\section{References}

[1] P. Ekman, W. V. Friesen, Nonverbal leakage and clues to deception, Journal for the Study of Interpersonal Processes 32 (1969) 88-106.
[2] P. Ekman, W. V. Friesen, Constants across cultures in the face and emotion, Journal of personality and social psychology 17(2) (1971) 124.

[3] P. Ekman, Lie catching and microexpressions, The philosophy of deception (2009) 118-133.

[4] S. Porter, L. ten Brinke, Reading between the lies identifying concealed and falsified emotions in universal facial expressions, Psychological Science 19(5) (2008) 508-514.

[5] M. G. Frank, M. Herbasz, K. Sinuk, A. Keller, A. Kurylo, C. Nolan, I see how you feel: Training laypeople and professionals to recognize fleeting emotions, in: Annual meeting of the International Communication Association, Sheraton New York, New York City, NY, 2009, pp.--.

[6] M. O'Sullivan, M. G. Frank, C. M. Hurley, J. Tiwana, Police lie detection accuracy: The eect of lie scenario, Law and Human Behavior 33(6) (2009) 530-538.

[7] M. G. Frank, C. J. Maccario, V. Govindaraju, Protecting Airline Passengers in the Age of Terrorism, ABC-CLIO, 2009, pp. - .

[8] P. Ekman, W. V. Friesen, Facial action coding system, Consulting Psychologists Press, 1978.

[9] W.-J. Yan, S.-J. Wang, G. Zhao, X. Li, Y.-J. Liu, Y.-H. Chen, X. Fu, CASME II: An improved spontaneous micro-expression database and the baseline evaluation, PLoS ONE 9 (2014) e86041.

[10] C. Anitha, M. Venkatesha, B. S. Adiga, A survey on facial expression databases, Int. Journal of Eng. Science and Tech. 2 (10) (2010) 5158-5174.

[11] M. Shreve, S. Godavarthy, V. Manohar, D. Goldgof, S. Sarkar, Towards macro-and micro-expression spotting in video using strain patterns, in: Applications of Computer Vision (WACV), 2009, pp. 1-6.

[12] S. Polikovsky, Y. Kameda, Y. Ohta, Facial micro-expressions recognition using high speed camera and 3d-gradient descriptor, in: Crime Detection and Prevention (ICDP 2009), 3rd Int. Conf. on, 2009, pp. 1-6.

[13] P. Ekman, Emotions revealed: Recognizing faces and feelings to improve communication and emotional life, Macmillan, 2007.

[14] X. Li, T. Pfister, X. Huang, G. Zhao, M. Pietikainen, A spontaneous micro-expression database: Inducement, collection and baseline, in: Automatic Face and Gesture Recognition, 2013, pp. $1-6$.

[15] W. J. Yan, Q. Wu, Y. J. Liu, S. J. Wang, X. Fu, Casme database: a dataset of spontaneous micro-expressions collected from neutralized faces, in: IEEE International Conference and Workshops In Automatic Face and Gesture Recognition, 2013, pp. 1-7.

[16] W. Qu, F., S. J., W. J. Yan, H. Li, S. Wu, X. Fu, $\mathrm{CAS}(\mathrm{ME})^{2}$ : A database for spontaneous macro-expression and micro-expression spotting and recognition, IEEE Transactions on Affective Computing.

[17] T. F. Cootes, C. J. Taylor, D. H. Cooper, J. Graham, Active shape models-their training and application, Computer Vision and Image Understanding 61(1) (1995) 38-59.

[18] A. Goshtasby, Image registration by local approximation methods, Image and Vision Computing 6(4) (1988) 255-261.

[19] G. Zhao, M. Pietikainen, Dynamic texture recognition using local binary patterns with an application to facial expressions, Pattern Analysis and Machine Intelligence, IEEE Transactions 29 (6) (2009) 915-928.

[20] J. A. Suykens, J. Vandewalle, Least squares support vector machine classifiers, Neural processing letters 9(3) (1999) 293-300.

[21] Y. Wang, J. See, R. C. W. Phan, Y. H. Oh, LBP with six intersection points: Reducing redundant information in lbp-top for micro-expression recognition, in: Computer Vision-ACCV, 2014, pp. 525-537.

[22] X. Huang, S. J. Wang, G. Zhao, M. Piteikainen, Facial microexpression recognition using spatiotemporal local binary pattern with integral projection, in: ICCV Workshops, 2015, pp. $1-9$.

[23] X. Huang, G. Zhao, X. Hong, W. Zheng, M. Pietikinen, Spontaneous facial micro-expression analysis using spatiotemporal 
completed local quantized patterns, Neurocomputing 175 (2016) $564-578$.

24] S. T. Liong, R. C.-W. Phan, J. See, Y. H. Oh, K. Wong, Optical strain based recognition of subtle emotions, in: International Symposium on Intelligent Signal Processing and Communication Systems, 2014, pp. 180-184.

[25] S.-T. Liong, J. See, R. C.-W. Phan, A. C. Le Ngo, Y.-H. Oh, K. Wong, Subtle expression recognition using optical strain weighted features, in: Asian Conference on Computer Vision, Springer, 2014, pp. 644-657.

[26] Y. H. Oh, A. C. Le Ngo, J. See, S. T. Liong, R. C. W. Phan, H. C. Ling, Monogenic riesz wavelet representation for microexpression recognition, in: Digital Signal Processing, IEEE, 2015, pp. 1237-1241

[27] S. Wang, W. Yan, X. Li, G. Zhao, C. Zhou, X. Fu, M. Yang, J. Tao, Micro-expression recognition using color spaces, IEEE Transactions on Image Processing 24(12) (2015) 6034-6047.

[28] Y.-J. Liu, J.-K. Zhang, W.-J. Yan, S.-J. Wang, G. Zhao, X. Fu, A main directional mean optical flow feature for spontaneous micro-expression recognition, IEEE Transactions on Affective Computing 7 (4) (2016) 299-310.

[29] A. C. Le Ngo, J. See, R. C. W. Phan, Sparsity in dynamics of spontaneous subtle emotions: Analysis \& application, IEEE Transactions on Affective Computing (2016) To appear.

[30] F. Xu, J. Zhang, J. Wang, Microexpression identification and categorization using a facial dynamics map, IEEE Transactions on Affective Computing (2016) In press.

[31] Z. Zhou, G. Zhao, Y. Guo, M. Pietikainen, An image-based visual speech animation system, IEEE Trans. on Circuits and Systems for Video Technology 22 (10) (2012) 1420-1432.

[32] X. Ben, P. Zhang, R. Yan, M. Yang, G. Ge, Gait recognition and micro-expression recognition based on maximum margin projection with tensor representation, Neural Computing and Applications 27(8) (2016) 2629-2646.

[33] P. Zhang, X. Ben, R. Yan, C. Wu, C. Guo, Micro-expression recognition system, Optik-International Journal for Light and Electron Optics 127(3) (2016) 1395-1400.

[34] S. Wang, W. J. Yan, G. Zhao, X. Fu, C. Zhou, Micro-expression recognition using robust principal component analysis and local spatiotemporal directional features, in: ECCV Workshops, 2014, pp. 325-338.

35] S. J. Wang, W. J. Yan, T. Sun, G. Zhao, X. Fu, Sparse tensor canonical correlation analysis for micro-expression recognition, Neurocomputing 214 (2016) 218-232.

[36] A. Moilanen, G. Zhao, M. Pietikainen, Spotting rapid facial movements from videos using appearance-based feature difference analysis, in: International Conference on Pattern Recognition (ICPR), 2014, pp. 1722-1727.

[37] A. K. Davison, M. H. Yap, C. Lansley, Micro-facial movement detection using individualised baselines and histogram-based descriptors, in: Systems, Man, and Cybernetics (SMC), 2015, pp. 1864-1869.

[38] S. J. Wang, S. Wu, X. Qian, J. Li, X. Fu, A main directiona maximal difference analysis for spotting facial movements from long-term videos, Neurocomputing 230 (2017) 382-389.

[39] X. Li, X. Hong, A. Moilanen, X. Huang, T. Pfister, G. Zhao, M. Pietikinen, Reading hidden emotions: Spontaneous micro-expression spotting and recognition, arXiv preprint arXiv:1511.00423.

[40] W. J. Yan, S. J. Wang, Y. H. Chen, G. Zhao, X. Fu, Quantifying micro-expressions with constraint local model and local binary pattern, in: Computer Vision-ECCV workshop, 2014, pp. 296305.

[41] S.-T. Liong, J. See, K. Wong, A. C. Le Ngo, Y.-H. Oh, R. Phan, Automatic apex frame spotting in micro-expression database, in: Pattern Recognition (ACPR), 2015 3rd IAPR Asian Conference on, 2015, pp. 665-669.

[42] S. T. Liong, J. See, K. Wong, R. C. W. Phan, Automatic microexpression recognition from long video using a single spotted apex, in: Asian Conference on Computer Vision, 2016, pp. 345360 .
[43] A. C. Le Ngo, S. T. Liong, J. See, R. C. W. Phan, Are subtle expressions too sparse to recognize?, in: Digital Signal Processing (DSP), 2015, pp. 1246-1250.

[44] P. Ekman, Facial expression and emotion., American psychologist 48 (4) (1993) 384.

[45] A. Esposito, The amount of information on emotional states conveyed by the verbal and nonverbal channels: Some perceptual data, in: Progress in nonlinear speech processing, Springer, 2007, pp. 249-268.

[46] A. Asthana, S. Zafeiriou, S. Cheng, M. Pantic, Robust discriminative response map fitting with constrained local models, in: Proc. of the IEEE Conf. on Computer Vision and Pattern Recognition, 2013, pp. 3444-3451.

[47] D. Fleet, Y. Weiss, Optical flow estimation, Springer, 2006.

[48] C. Zach, T. Pock, H. Bischof, A duality based approach for realtime TV-L1 optical flow, Pattern Recognition (2007) 214223.

[49] J. C. Simof, T. J. R. Hughes, Computational Inelasticity, Springer, 2008.

[50] R. Chaudhry, A. Ravichandran, G. Hager, R. Vidal, Histograms of oriented optical flow and binet-cauchy kernels on nonlinear dynamical systems for the recognition of human actions, in: Computer Vision and Pattern Recognition, 2009, pp. 19321939.

[51] A. C. Le Ngo, R. C.-W. Phan, J. See, Spontaneous subtle expression recognition: Imbalanced databases and solutions, in: Asian Conference on Computer Vision, Springer, 2014, pp. 3348.

[52] S.-T. Liong, J. See, R. C.-W. Phan, Y.-H. Oh, A. C. Le Ngo, K. Wong, S.-W. Tan, Spontaneous subtle expression detection and recognition based on facial strain, Signal Processing: Image Communication 47 (2016) 170-182. 